Discussion Paper No. 834

\title{
COMPETITIVE TARGETED MARKETING
}

Hang-Hyun Jo

Jeong Yoo Kim

March 2012

The Institute of Social and Economic Research

Osaka University

6-1 Mihogaoka, Ibaraki, Osaka 567-0047, Japan 


\title{
Competitive Targeted Marketing
}

\author{
Hang-Hyun Jo \\ Jeong-Yoo Kim * \\ Korea Institute for Advanced Study \\ Kyung Hee University
}

March 1, 2012

\begin{abstract}
In this paper, we consider two firms diffusing incompatible technologies and their decision of consumer targeting. The technology adoption is made in two steps. First, once the firms sell their products to their respective targeted consumer, the technology is diffused successively by word-of-mouth communication from the initial consumer to other consumers linked along the network. Then, in the second step, each consumer imitates the technology the neighbors use which fares better, and through this process of imitation, the technology distribution keeps evolving until it reaches the long-run steady state. We demonstrate that the early entrant chooses the minmax location when firms are myopic in the sense that they do not take the imitation possibility into account. If firms consider the possibility of imitation, the best target will tend towards a hub, although the minmax principle in general keeps valid in the sense that it should be the minmax location after considering imitation.
\end{abstract}

Keywords: Information Diffusion, Technology Adoption, Network Externalities, Compatibility, Learning, Evolution, Targeted Marketing

*This research was begun when the second author was visiting ISER at Osaka University. W are very grateful to Masaki Aoyagi for his hospitality during our stay. Corresponding author: Department of Economics, Kyung Hee University, 1 Hoegi-dong, Dongdaemun-gu, Seoul 130-701, Republic of Korea, Tel: +82-2-961-0986, Fax: +82-2-966-7426, Email: jyookim@khu.ac.kr 


\section{Introduction}

Technologies are evolving. An old technology soon faces competition by the emergence of a new technology. It may be replaced by the new superior technology or they may coexist for a while. If the competing technologies are not compatible with each other, a consumer should be concerned about how many neighbors share the technology in deciding which technology to adopt. The more neighbors use the technology, the higher utility a consumer usually gets by adopting it. This is called network externality. ${ }^{1}$

In the description above, special attention should be paid to the word "neighbor." If a consumer meets only a limited set of the whole population, the total number of users adopting the same technology is not meaningful to him. He only needs to care about the technologies adopted by his neighbors, that is, those whom he interacts with. This implies that the structure of the network among consumers in which they interact with each other is an important factor to be considered in deciding which technology to adopt in the presence of network externalities. However, the explicit structure of the consumer network has been largely ignored in literature on technology adoption.

The network structure is crucial not only to consumers but also to the firms sponsoring the technologies. As long as the technologies are diffused by word-of-mouth communication between consumers along the consumer network, it is important for a firm to target a right consumer so as to maximize its market share.

In this paper, we consider two competing firms sponsoring incompatible technologies and their decision of consumer targeting. We also consider the subsequent diffusion and evolution of technologies. The technology adoption is made in two steps. First, once the firms sell their products to their respective targeted consumer, the technology is diffused by word-of-mouth communication from the initial consumer to other consumers linked along the network subsequently. Then, in the second step, each consumer imitates the technology the neighbors use which fares better, and through this process of imitation, the technology distribution keeps evolving until it reaches the long-run steady state.

The economists' interest in the consumer network has begun only recently. Jun and Kim (2008) seems the first paper to consider the consumer network explicitly. They consider the monopolist's problem of choosing the referral fee to provide consumers incentives enough to make the information about its products spread widely. Subsequently, Jun et al. (2006a,

\footnotetext{
${ }^{1}$ This concept has been actively explored in the context of technology compatibility (standardization) by economists since David (1985), Farrell and Saloner (1985) and Katz and Shapiro (1985).
} 
2006b) consider similar problems in small world networks. Also, Kim (2010) considers the targeting decision of a monopolist whose objective is to maximize its discounted sum of values generated from the information diffusion. Since a single decision maker is involved in the paper, there is no strategic aspect to be considered. This paper is an extension of Kim (2010) into a duopoly setting. ${ }^{2}$

Recently, Duan et al. (2005) investigate a targeting strategy for the spread of opinions, diseases, new ideas, technological innovations, etc. in scale-free social networks. They are mainly interested in how to pick out a given multiple number of agents as initial targets in order to launch a spreading process efficiently, and propose a strategy of targeting agents with most connections (hubs) as initial adopters, so-called hub strategy. In this paper, we investigate the targeting strategies of competing technologies and the resulting technology diffusion in an agent-based model by focusing more on the micro motivation of agents, ${ }^{3}$ and establish the minmax principle in competitive targeting. If two firms with sequentially developed technology compete in targets, the first firm does not target naively as if he were a single player, but chooses its target by considering its effect on the second firm's decision. The second firm's decision is naturally affected by the first firm's targeting decision. Then, the optimum for the first firm is to choose the minmax node, i.e., the node to minimize the maximum of the rival's market share. The main insights of this paper, however, can be straightforwardly applied to the opinion formation and others, as we will argue later.

Some could be learned from the previous works on the epidemic spreading and the efficient immunization strategies on the complex networks. ${ }^{4}$ For example, the targeted immunization strategy turns out to be more efficient to prevent the disease spreading than the random immunization strategy on the scale-free networks. Duan et al. (2005) modified the strategy by targeting the higher degree nodes with higher probability. While all those works consider the diffusion of one kind of technology (monopoly), we take into account the competition between two incompatible technologies, which makes the problem qualitatively different. On the other hand, Newman (2005) studied the case in which after one pathogen spreads first on

\footnotetext{
${ }^{2}$ We found the paper by Galeotti and Goyal (2009) which also addressed a problem of targeting in the consumer network. However, this paper mainly considers monopoly targeting, not competitive targeting.

${ }^{3}$ Many opinion dynamics models studied by the physicists usually simply assume the compromise process between the agents, as in the voter model (See, for example, Liggett [1999]) and the Sznajd model See Sznajd [2000, 2005]) without paying enough attention to the agents' economic motivation. This might lead to misunderstanding when applied to economic phenomena.

${ }^{4}$ For example, see Pastor-Satorras and Vespignani (2002), Havlin et al. (2003) and Jo et al. (2006).
} 
a network, then the other pathogen spreads over the remaining population of that network. Our paper is also distinguished from the work in the sense that we consider the concurrent diffusions of two sequentially developed technologies.

The paper is organized as follows. In Section 2, we describe the model and provide its economic rationale. In Section 3, we consider the firms' targeting decision and the consequent technology diffusion process when the network is a tree. In Section 4, we discuss the case of more general networks and provide some simulation results. Section 5 contains the conclusion.

\section{Model and Economic Rationale}

We consider only the connected network consisting of $N$ nodes. Each node represents a consumer, who is a potential technology user. Two firms are going to introduce two technologies which are incompatible to each other. We will call the technology introduced by firm $A$ and $B$ technology $A$ and $B$ respectively. The firms can diffuse their technology by choosing a node as the starting consumer. To avoid uncertainty due to simultaneity, we favor firm $A$ by giving him the first mover advantage. That is, we assume that firm $A$ first chooses a node $i_{A}$ to diffuse its technology and then firm $B$ chooses a target node $i_{B}$. Considering the reality that two competing technologies are rarely introduced simultaneously, it is a realistic assumption.

The subsequent interaction among technology users consists of two stages, each of which again consists of multi-periods. The first stage is the technology diffusion stage. Once a node adopts a technology $\alpha \in\{A, B\}$, he passes the information on the technology to all his neighbors in the period. Then, the neighbors in turn inform the technology to all their neighbors in the next period, and so one. This diffusion process is proceeded until no one remains uninformed. In other words, given the initial targets of two technologies $\left(i_{A}, i_{B}\right)$, the technology of node $i$ will be $\alpha_{i}=\arg \min _{\alpha \in\{A, B\}} d\left(i, i_{\alpha}\right)$, where $d(i, j)$ is the shortest path length between nodes $i$ and $j$. If node $i$ receives the information on the two technologies at the same time, i.e. $d\left(i, i_{A}\right)=d\left(i, i_{B}\right)$, we assume that he adopts the technology $A$ with probability $p \in[0,1] .^{5}$

The second stage is the technology imitation stage. Once the diffusion of the technologies

\footnotetext{
${ }^{5}$ More realistically one may assume that the probability depends positively on the number of neighbors using the same technology. However, for the complexity reason, we do not consider the case in this paper.
} 
is completed, each pair of nodes linked with each other play the $2 \times 2$ game in which both users obtain a positive payoff $a$ ( $b$ respectively) if they choose the same technologies $A$ ( $B$ respectively) and both obtains zero otherwise. ${ }^{6}$ The structure implies that the two technologies exhibit network externalities. ${ }^{7}$ After playing the $2 \times 2$ game, each user compares the average payoff of his $A$-neighbors and $B$-neighbors including his own payoff, and imitates the technology yielding the higher average payoff. After the transient periods the system will be stationary, resulting in the stationary population distribution of users of each technology.

The imitation dynamics is described precisely. Let $t=0$ be the period at which technology diffusion is completed and let $N^{A}(t)$ be the number of users adopting technology $A$ at period $t=0,1,2, \cdots$. Then $N^{B}(t)=N-N^{A}(t)$. Let us denote the sets of a node $i$ 's $A$-neighbors and $B$-neighbors as $N_{i}^{A}$ and $N_{i}^{B}$ respectively. Then $N_{i}=N_{i}^{A} \cup N_{i}^{B}$. From $k_{i}^{A} \equiv\left|N_{i}^{A}\right|$ and $k_{i}^{B} \equiv\left|N_{i}^{B}\right|$, the node $i$ 's degree is $k_{i}=k_{i}^{A}+k_{i}^{B}$. If the node $i$ adopts technology $A$, i.e. $\alpha_{i}=A$, his payoff is given by $\pi_{i}=\frac{k_{i}^{A}}{k_{i}} a$. Similarly, if $\alpha_{i}=B$, then $\pi_{i}=\frac{k_{i}^{B}}{k_{i}} b$. Only the node having both $A$-neighbors and $B$-neighbors has the chance to switch its technology. If $\alpha_{i}=A$, the node $i$ switches to $B$ if and only if

$$
\frac{\sum_{l \in N_{i}^{A} \cup\{i\}} \pi_{l}}{k_{i}^{A}+1}<\frac{\sum_{l \in N_{i}^{B}} \pi_{l}}{k_{i}^{B}},
$$

and similarly, if $\alpha_{j}=B$ for node $j$, he switches to $A$ if and only if

$$
\frac{\sum_{l \in N_{j}^{A}} \pi_{l}}{k_{j}^{A}}>\frac{\sum_{l \in N_{j}^{B} \cup\{j\}} \pi_{l}}{k_{j}^{B}+1} .
$$

By applying the Bethe approximation we briefly analyze how the imitation dynamics depends on the degrees of involved nodes. Consider a node $i$ with $\alpha_{i}=A$ is connected with

\footnotetext{
${ }^{6}$ An earlier evolutionary approach to the technology adoption game can be found in Kandori and Rob (1998). However, in their model, a pair of players playing the $2 \times 2$ game are randomly matched from the whole population, not locally matched on a network. Thus, the set of players each player is expected to play the $2 \times 2$ game is the same in their model, while each player plays the game with a different set of players in our model. Another difference is that the proportion of each type evolves according to the best response dynamics in their model, while it evolves according to the imitation dynamics. Consequently, in this model, if the network is complete, each player will play with the same group of players as in their model, but the imitation process will be different from theirs.

${ }^{7}$ This payoff structure can be used for opinion formation. Suppose there are two conflicting views and imagine that the interaction between two individuals with similar opinions reinforces the belief of each other, while the (one-time) interaction between individuals with differing opinions yields only quarrel with no utility. If one view, say $A$, is more convincing than the other, say $B$, then the former will have a stronger reinforcing effect in the sense that $a>b$.
} 
$k_{i}^{A} A$-neighbors and $k_{i}^{B} B$-neighbors. We assume for simplicity that each $A$-neighbor of node $i$, say $l$, has $k_{l}$ neighbors and those next nearest neighbors adopt either $A$ or $B$ with the probabilities $\rho=\frac{N^{A}}{N}$ and $1-\rho$. Similarly we assume that each $B$-neighbor of node $i$, say $j$, has $k_{j}$ neighbors and those next nearest neighbors adopt one of technologies in the same way. Since $\pi_{i}=\frac{k_{i}^{A}}{k_{i}} a, \pi_{l}=\frac{\left(k_{l}-1\right) \rho+1}{k_{l}} a$, and $\pi_{j}=\frac{\left(k_{j}-1\right)(1-\rho)}{k_{j}} b$, the condition (??) for switching from $A$ to $B$ yields

$$
\frac{k_{i}^{A} \pi_{l}+\pi_{i}}{k_{i}^{A}+1}<\frac{k_{i}^{B} \pi_{j}}{k_{i}^{B}}
$$

i.e.,

$$
\frac{k_{i}^{A}}{k_{i}^{A}+1}\left[\frac{1}{k_{i}}+\frac{\left(k_{l}-1\right) \rho+1}{k_{l}}\right] a<\frac{k_{j}-1}{k_{j}}(1-\rho) b .
$$

Combined with the condition from (??), one can roughly say node $i$ is more likely to invade or less likely to be invaded as the number of the same type neighbors, $k_{i}^{A}$, is larger and the degree of the opposite type neighbors, $k_{j}$, is smaller. Let a link connecting $i$ with $\alpha_{i}=A$ and $j$ with $\alpha_{j}=B$ on a tree network be the unique bridge between $A$-cluster and $B$-cluster, implying that without that link, the tree will be split into two clusters of each own type. Then the condition (??) reduces to

$$
\frac{k_{i}^{2}-1}{k_{i}^{2}} a<\frac{k_{j}-1}{k_{j}} b .
$$

Since $k_{i}=k_{i}^{A}+1$, the above analysis is clarified in this case.

The objective of each firm is to maximize the number of its technology users by targeting its starting user optimally. For the subsequent analysis, we assume that there is no discount in the profit of firms. ${ }^{8}$ This assumption implies that firms care only about the stationary distribution, that is, cares only about the profit in the long-run future state with ignoring the profit in the transient periods. To isolate the targeting decisions from other strategic effects, we are also implicitly assuming that there is no price competition. If each firm can choose its price as well as its target, the imitation decision of consumers will depend on the prices as well. Then, the second firm may try to invade consumers using the rival's technology by charging a lower price than the rival. Thus, there will be a strategic interaction between

\footnotetext{
${ }^{8}$ The assumption of no discounting might be challenged. If the evolution phase is too long, firms may care about the profits realized before the steady state is reached. However, if we consider discounting explicitly, the optimal targeting decision will depend on the discounting factor as well, which complicates the prediction. See Kim (2010) for the the targeting decision of a monopolist who takes discounting into account.
} 
firms in pricing. Although this is an interesting economic problem, it is beyond the scope of this paper.

\section{Consumer Targeting in a Tree}

The targeting decision of the firms induces a partition of users, each of which uses the same technology as a consequence of information diffusion. Let $\mathcal{P}$ and $\mathcal{P}^{\prime}$ be two partitions. Note that a partition is entirely determined by the set of users adopting technology $A$. Accordingly, let $A(\mathcal{P})$ be the users adopting technology $A$ in $\mathcal{P}$.

A firm's preference over partitions is denoted by $\succsim$. That is, $\mathcal{P} \succsim_{A} \mathcal{P}^{\prime}$ implies that firm $A$ prefers $\mathcal{P}$ to $\mathcal{P}^{\prime}$, and $\mathcal{P} \sim_{A} \mathcal{P}^{\prime}$ implies that firm $A$ is indifferent between $\mathcal{P}$ and $\mathcal{P}^{\prime}$. By the constant-sum nature, i.e., $N^{A}+N^{B}=N, \mathcal{P} \succsim_{A} \mathcal{P}^{\prime}$ implies that $\mathcal{P}^{\prime} \succsim_{B} \mathcal{P}$.

We consider two cases. One is the case that firms are myopic so that they do not consider the possibility of technology imitation in their targeting decisions. The other is the case of sophisticated (far-sighted) firms who make targeting decisions by considering the subsequent imitation process.

In this section, the analysis will be restricted to tree networks. Consumer targeting in more general networks will be discussed in the next section.

\subsection{Myopic Firms}

Given the sequential nature of targeting, we first consider the decision of the entrant firm $B$. Let $i_{B}^{*}(i)$ be the best response of firm $B$ to $A$ 's target $i$.

Lemma 1 For any $i_{A}=i, d\left(i, i_{B}^{*}\right)=1$.

This lemma implies that the targets of the firms must be minimally differentiated. The intuition is that a firm always prefers being more closely located to the opponent in order to secure more customers.

Proposition 1 Given any $i_{A}=i,\left\{i_{B}^{*}(i)\right\}=\arg \max _{j \in N_{i}}\left|S_{j}^{i}\right|$.

Here $S_{j}^{i}$ denotes a set of node $j$ 's successors against node $i$.

Now, consider incumbent $A$ 's decision. Letting $i_{A}^{*}$ be the optimal target, we have the following proposition. 
Proposition $2\left\{i_{A}^{*}\right\}=\arg \min _{i \in N} \max _{j \in N_{i}}\left|S_{j}^{i}\right|$.

This proposition says that it is optimal for firm $A$ to take the minmax strategy. For example, in a line network with $N=2 m+1$ for some positive integer $m$ illustrated in Figure 1(a), $i_{A}^{*}=m+1$. Note that this target does not necessarily correspond to a hub, as illustrated in Figure 1(b). In Figure 1(b), firm $A$ will target node 5, although node 4 is the hub with the highest degree.

\subsection{Sophisticated Firms}

If firms are sophisticated, they choose their targets not simply by maximizing the current market share, but by taking the subsequent imitation process into account. Thus, it is not straightforward to compare their preference between two partitions. However, the following proposition simplifies the process of searching for the optimal target.

Proposition 3 Suppose $i_{A}=i_{1}, i_{2}$ induce $\mathcal{P}_{1}=\left\{A_{1}, B_{1}\right\}$ and $\mathcal{P}_{2}=\left\{A_{2}, B_{2}\right\}$ respectively. When firms are sophisticated, $A_{1} \supset A_{2}$ implies that $\mathcal{P}_{1} \succsim_{A} \mathcal{P}_{2}$.

Proposition 3 says that sophisticated firms prefer a larger installed base even if they take into account the possibility that the imitation process will lead to a different market share ultimately. Due to this proposition, it is easy to see that Lemma 1 still holds in the case of sophisticated firms. To see this, suppose $d\left(i, i_{B}^{*}(i)\right) \geq 2$ for some node $i$. Now, alternatively take $i_{B}=j$ where $d(i, j)=1$ and $j \in q_{i, i_{B}^{*}}$, where $q_{i, j}$ denotes a path from $i$ to $j$. Then, clearly, the partition induced by the latter choice is preferred by the entrant $B$. However, Proposition 1 does not hold any more. To see this, consider Figure 1(c). Assuming $a<b$, given that $i_{A}=6$, if firm $B$ enters $i_{B}=7$, technology $B$ cannot invade $i_{A}$ if $a>2 b / 3$. If firm $B$ enters $i_{B}=5$ instead, it can invade node 6 if $(2 b / 3<) a<8 b / 9$. This implies that it can be optimal for firm $B$ to enter node 5 even if $i_{B}^{*}(6)=7$ in the case of myopic firms. The main insight is that node 5 has more neighbors so as to be advantageous for invasion. A similar intuition will be provided for the targeting decision of firm $A$. Take the example of the network in Figure 1(d) and assume that the value of $a=1$ and $b=1.5-\epsilon$. If firms are myopic, $\left(i_{A}^{*}, i_{B}^{*}\right)=(9,4)$ as we showed. But, then, nodes 8 and 9 of type $A$ are invaded by type $B$. Ultimately, the market share of firm $A$ will end up with $6 / 15$. However, if firm $A$ targets node 4 , node 4 of type $A$ is not invaded and the resultant market share of firm $A$ is $7 / 15$. Thus, node 4 is preferable to firm $A$. The intuition is as follows. For a node of firm $A$ not to be invaded, it is better that the opponent node has less neighbors. Note that node 4 
has a large number of neighbors making him faring very well regardless of the technology he chooses. This implies that if firm $A$ enters node 9 , it will be very likely to be invaded. The upshot is that if an early entrant expects to face a new entrant with superior technology, it must keep the rival firm from entering the hub. Thus, it may be sometimes (not always) good to preempt such a hub. If the incumbent has the superior technology, it may prefer choosing the hub for a different reason that it is more advantageous for invasion.

The general conclusion is that firms want their target to have more neighbors and their opponent's target to have less neighbors in the invasion battle. More specifically, the firm with a superior technology prefers entering the hub to invade neighbors more easily, while the firm with an inferior technology would rather choose the node whose rival node has less neighbors in order to be less vulnerable to the invasion. ${ }^{9}$ However, this does not imply that the sophisticated firm always prefers the hub with the highest degree to the myopic optimal targets. If the non-hub myopic targets are robust against invasion, there would be no reason to give them up in order to preempt hub targets.

Generally, we can identify the optimal target of the two firms in the sophisticated case. For given $i$, find $i_{B}^{*}(i)$ by the node $j \in N_{i}$ that maximizes $\left|\tilde{S}_{i}^{j}\right|$ where $\tilde{S}_{i}^{j}$ is the set of $B$ technology users after the imitation is completed. The node $j \in \arg \max \left|\tilde{S}_{i}^{j}\right|$ is an optimal response of firm $B$. Then, an optimal target of firm $A$ is $i_{A}^{*} \in \arg \min _{i \in N} \max _{j \in N_{i}}\left|\tilde{S}_{i}^{j}\right|$.

\section{Discussion on Barabási-Albert Scale-Free Networks}

We will briefly discuss the case of more general networks possibly containing a cycle. First of all, Lemma 1 is not valid any more. For example, consider a circle. Once firm $A$ chooses a node in a circle, any other node is optimal to firm $B$. In any case, the two firms split the consumers equally in the technology diffusion stage. Suppose $d\left(i_{A}, i_{B}\right)=2$ in a circle graph with $N=5$. Then, by moving closer to $i_{A}$, firm $B$ may secure the node between them but it loses other nodes in the opposite direction. In this case, note that node $j$ is not critical in the communication between $i_{A}$ and $k \neq i_{A}, i_{B}, j$. The fact that the path between two nodes $i_{A}$ and $i_{B}$ is not unique causes Lemma 1 to fail. This observation forces us to search for firm

\footnotetext{
${ }^{9} \mathrm{We}$ are saying that the reason for the hub preference is either defensive (for an inferior firm) or offensive (for a superior firm), but this distinction is just an overall tendency, since the possibility of invasion depends on the number of neighbors as well as the quality of technology. In other words, an inferior firm might prefer a hub-like node for an offensive reason. This means that the two effects actually coexist. It is true, too, for the case that the two technologies have the same quality, i.e., $a=b$.
} 
$B$ 's optimal response to $i_{A}$ not only from the neighbors but globally from the population. More specifically, for any (nonterminal) node $i$ and for each $j \in N_{i}$, one can induce the probability distribution over the technology distribution as the result of the naive diffusion based on $p$, since the existence of a cycle makes it possible for two technologies to arrive at a node simultaneously. Then, compute the expected number of successors $\hat{S}_{i}^{j}$. Then, an optimal target is $i_{A}^{*} \in \arg \min _{i \in N} \max _{j \in N_{i}}\left|\hat{S}_{i}^{j}\right|$, which will be called the extended $\operatorname{minmax}$ target.

We provide the simulation results in Figure 2 for $N=51$. To generate networks, we use two Barabási-Albert scale-free networks. ${ }^{10}$ Two kinds of networks are considered; a tree which are obtained by connecting each new node to only one existing node, and a general network with cycles which are obtained by connecting each new node to two existing nodes.

Given the generated networks, we first simulate the technology diffusion process. Take any pair of nodes and assign $A$ and $B$ to them respectively. In case of trees, it suffices to take any pair of adjacent nodes. Then assign $A$ to other nodes if they are closer to the initial $A$ node and assign $B$ to nodes if they are closer to the initial $B$ node. If the distances to the initial $A$ and the initial $B$ are equal, just assign $A$ (by assuming $p=1$ ). Then, we simulate the second stage technology imitation process by the random sequential updating method. That is, at each time step of imitation process one randomly chosen node can be invaded by its neighbors of different type. This randomness of choosing a node forces us to take the average of the final market share. Thus, to find the minmax strategy of sophisticated firms we compare the averaged market shares.

In Figures $2(\mathrm{a})$ and $(\mathrm{b})$, the optimal targets are $\left(i_{A}, i_{B}\right)=(3,1)$ and $(3,7)$ when firms are myopic and sophisticated, respectively. Note that $k_{7}=5$ is larger than $k_{1}=3$. This supports our main insight that sophisticated firms tend to prefer nodes with higher degree. In Figures 2(c) and (d), interestingly, the optimal targets for myopic firms are $(4,1)$ but the optimal ones for sophisticated firms are reversed to $(1,4)$. Firm A, who is the earlier entrant, can foresee that node 4 which is its optimal target in the myopic case will be invaded and its resulting market share will be much lower than the rival's market share. By preempting node 1 instead of node 4, it can increase the number of its users from 11.22 to 11.44.

In Figure 3, we illustrate the tendency of sophisticated firms towards nodes with higher degree more dramatically for a larger population $N=501$. It shows a correlation between the degree of the extended minmax target and the market share. Note that this result should

\footnotetext{
${ }^{10}$ See Barabási and Albert (1999).
} 
not be taken for granted, since it incorporates the concomitant effect of firm $B$ 's optimal response to firm $A$ 's extended minmax target.

Figure 4 illustrates the correlation between the relative quality $b / a$ and the market share $N^{A}$. It shows that the market share of technology $A$ tends to decrease with $b / a$. This is not surprising, since a technology will lose its market share more as the competing technology has a higher quality. However, the figure also shows that the distribution of $N^{A}$ becomes more dispersed as $b / a$ increases, which is surprising. This is one consequence of the firstmover advantage. One clear aspect of the first-mover advantage is that $N^{A}$ is much larger than a half of the population (250) when $a=b$. Because of the severe advantage, the first mover's market share will mostly stay above 250 when $b / a \leq 1 / 2$. This is the main reason why the distribution of $N^{A}$ is so concentrated for low values of $b / a$. This is because high assortativity weakens the first mover advantage.

Figure 5 shows the relationship between the degree of sophistication $s$ and the market share $N^{A}$. By the degree of sophistication, we mean how far the firms foresee. For two extreme cases, the myopic case corresponds to the case that $s=0$ and the sophisticated case corresponds to the case that $s=50$. The horizontal line $s$ of Figure 5 represents how many rounds of the imitation process firms consider in deciding their target. The figures show that $N^{A}$ changes monotonically with $s$. Note that there are two kinds of advantages in this model; the quality advantage and the first-mover advantage. If $a \geq b$, technology $A$ is clearly advantageous, ${ }^{11}$ while technology $B$ is if $b \gg a$ since its quality advantage dominates the second-mover disadvantage. The advantages will become more stark as the firms get more sophisticated (smart). Thus, as $s$ gets higher, $N^{A}$ increases monotonically if $a \geq b$ and decreases monotonically if $a \ll b$. This is the case for both assortative and disassortative networks while the tendency on assortative networks is weaker than on disassortative networks. It is again because high assortativity weakens the first mover advantage. We also find an exceptional case in Figure 5(c), where $N^{A}$ increases slightly even when $b / a=1.4$ for $r=0.3$. This overall result extends the result of Duan et al. showing a positive relationship between the identification ability and the contagion rate. In this paper, the degree of sophistication is a counterpart for the identification ability and the market share is one for the contagion rate. Figure 5 tells us that the result of Duan et al. is carried over to the case of competitive targeting in the sense that the invasion rate of

\footnotetext{
${ }^{11}$ If $a=b$, technology $A$ has no quality advantage, but still has the net advantage because it is the first mover.
} 
the advantageous technology is higher as the firms are able to foresee farther.

\section{Conclusion}

In this paper, we examined the optimal targeting decisions of duopoly firms and obtained the result that they must pay attention to the minmax node, not simply to a hub due to the other competing firm's response. The general insight provided in this paper and the simulation method will help to better understand the evolution of competing technologies in a local interaction market with network externalities.

\section{Appendix}

Proof of Lemma 1: Let the induced technology partition be $\left(A^{*}, B^{*}\right)$. Suppose $d\left(i, i_{B}^{*}\right) \geq$ 2. Then, there must be $j \in q_{i, i_{B}^{*}}$ such that $d(i, j)=1$. Now, suppose firm $B$ chooses $j$ instead of $i_{B}^{*}$ as a target and let the induced partition be $\left(A^{\prime}, B^{\prime}\right)$. Since $j$ is critical in the communication between $i$ and $k$ for any $k \in B^{*}$ in the sense that $i$ and $k$ would be disconnected without $j$, it follows that $d(i, k)>d(j, k)$, implying that $k \in B^{\prime}$ for any $k \in B^{*}$. This in turn implies that $B^{*} \varsubsetneqq B^{\prime}$ since $j \in B^{\prime}$ but $j \notin B^{*}$. Thus, firm $B$ prefers $B^{\prime}$ to $B^{*}$. Contradiction.

Proof of Proposition 1: From Lemma 1, it is clear that $i_{B}^{*}(i) \in N_{i}$. It is trivial to see that it must generate the largest set of his successors.

Proof of Proposition 2: This is trivial, since the game is a constant-sum.

Proof of Proposition 3: Consider the set $A_{1} \backslash A_{2}$. Let $\tilde{A}_{2}=\left\{k \in A_{1} \backslash A_{2} \mid k \leftarrow A_{2}\right\}$. If $\tilde{A}_{2} \subsetneq A_{1} \backslash A_{2}$, it is clear that $A_{2} \cup \tilde{A}_{2} \subsetneq\left(A_{1} \backslash A_{2}\right) \cup A_{2}=A_{1}$. Then, it follows that $\mathcal{P}_{1} \succ_{A} \mathcal{P}_{2}$, since inequality (4) implies that $j \in B_{1}$ must be invaded for a larger set $A_{1}$ whenever it is invaded for a smaller set $A_{2} \cup \tilde{A}_{2}$. If $\tilde{A}_{2}=A_{1} \backslash A_{2}$, we have $\mathcal{P}_{1} \sim_{A} \mathcal{P}_{2}$ by the Markov property of the population distribution. 


\section{References}

[1] Albert, R., Barabási, A.-L., 2002. Statistical mechanics of complex networks, Reviews of Modern Physics 74, 47-97.

[2] Arenas, A., Diaz-Guilera, A., Perez, C.J., Vega-Redondo, F., 2000. Self-organized evolution in a socioeconomic environment, Physical Review E 61, 3466-3469.

[3] Barabási, A-L. and Albert, R., 1999, Emergence of Scaling in Random Networks, Science 286, 509-512.

[4] Boccaletti, S., Latora, V., Moreno, Y., Chavez, M., Hwang, D.-U., 2006. Complex networks: Structure and dynamics, Physics Reports 424, 175-308.

[5] Castellano, C., Fortunato, S., Loreto, V., 2009. Statistical physics of social dynamics, Reviews of Modern Physics 81, 591-646.

[6] David, P.A., 1985, Clio and the Economics of QWERTY, American Economic Review 75

[7] Cohen, R., Havlin, S., Ben-Avraham, D., 2003. Efficient Immunization Strategies for Computer Networks and Populations, Physical Review Letters 91, 247901.

[8] Duan, W., Chen, Z., Liu, Z., Jin, W., 2005. Efficient target strategies for contagion in scale-free networks, Physical Review E 72, 026133.

[9] Farrell, J. and G. Saloner, 1985, Standardization, Compatibility, and Innovation, Rand Journal of Economics 16, 70-83

[10] Galeotti, A. and S. Goyal, 2009. Influencing the Influencers: a Theory of Strategic Diffusion, Rand Journal of Economics 40, 509-532

[11] Guardiola, X., Diaz-Guilera, A., Perez, C.J., Arenas, A., Llas, M., 2002. Modeling diffusion of innovations in a social network, Physical Review E 66, 026121.

[12] Hołyst, J. A., Kacperski, K., Schweitzer, F., 2000. Phase transitions in social impact models of opinion formation, Physica A 285, 199-210. 
[13] Hołyst, J. A., Kacperski, K., 2001. Social impact models of opinion dynamics. In: Stauffer, D. (Eds.), Annual Reviews of Computational Physics IX, World Scientific Publishing Company, pp. 253-273.

[14] Jo, H.-H., Baek, S.K., Moon, H.-T., 2006. Immunization dynamics on a two-layer network model, Physica A 361, 534-542.

[15] Jun, T. and J.-Y. Kim, 2008. A Theory of Consumer Referral, International Journal of Industrial Organization 26, 662-278

[16] Jun, T., J.-Y. Kim, B. J. Kim and M. Y. Choi, 2006a. "Consumer Referral in a Small World Network." Social Networks 28: 232-246.

[17] Jun, T., J.-Y. Kim, B. J. Kim and M. Y. Choi, 2006b. Network Marketing on a Small World Network. Physica A 360, 493-504.

[18] Kandori, M. and R. Rob, 1998, Bandwagon Effects and Long Run Technology Choice, Games and Economic Behavior 22, 30-60

[19] Katz, M. and C. Shapiro, 1985, Network Externalities, Competition, and Compatibility, American Economic Review 75, 1985, 424-40

[20] Kim, J.-Y., 2010, Information Diffusion and $\delta$-Closeness-Centrality, Sociological Theory and Methods 25, 95-106

[21] Lambiotte, R., Redner, S., 2008. Dynamics of non-conservative voters, Europhysics Letters 82, 18007.

[22] Liggett, T.M., 1999. Stochastic Interacting Systems: Contact, Voter, and Exclusion Processes, Springer-Verlag, New York.

[23] Llas, M., Gleiser, P.M., Lopez, J.M., Diaz-Guilera, A., 2003. Nonequilibrium phase transition in a model for the propagation of innovations among economic agents, Physical Review E 68, 066101.

[24] Mantegna, R.N., Stanley, H.E., 2000. An Introduction to Econophysics, Cambridge University Press, Cambridge, England. 
[25] Newman, M.E.J., 2005. Threshold Effects for Two Pathogens Spreading on a Network, Physical Review Letters 95, 108701.

[26] Newman, M.E.J., 2003. The Structure and Function of Complex Networks, SIAM Review 45, 167-256.

[27] Newman, M.E.J., 2002. Assortative Mixing in Networks, Physical Review Letters 89, 208701.

[28] Pastor-Satorras, R., Vespignani, A., 2002. Immunization of complex networks, Physical Review E 65, 036104.

[29] Schweitzer, F., Behera, L., 2009. Nonlinear voter models: the transition from invasion to coexistence, The European Physical Journal B 67, 301-318.

[30] Shao, J., Havlin, S., Stanley, H.E., 2009. Dynamic Opinion Model and Invasion Percolation, Physical Review Letters 103, 018701.

[31] Stauffer, D., 2005. Sociophysics simulations II: opinion dynamics. In: Garrido, P.L., Marro, J., Munoz, M.A. (Eds.), Modeling Cooperative Behavior in the Social Sciences, AIP Conf. Proc. No. 779, AIP, Melville, NY, pp. 56-68.

[32] Sznajd-Weron, K., 2005. Sznajd model and its applications, Acta Physica Polonica B $36,2537-2547$.

[33] Sznajd-Weron, K., Sznajd, J., 2000. Opinion evolution in closed community, International Journal of Modern Physics C 11, 1157-1165. 
(a)

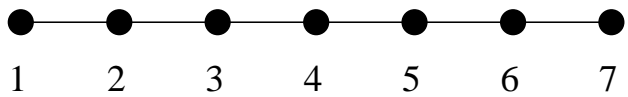

(b)

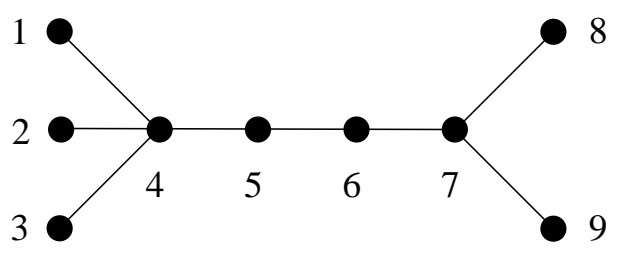

(c)

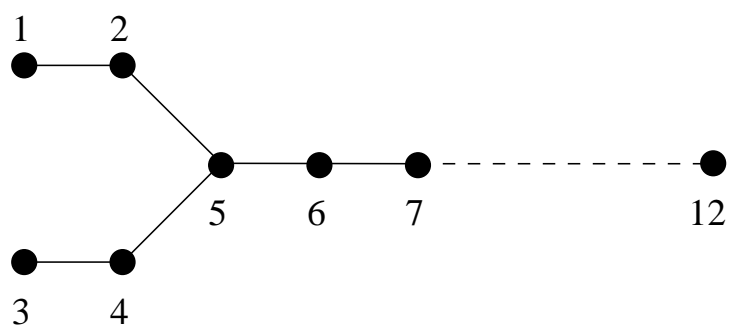

(d)

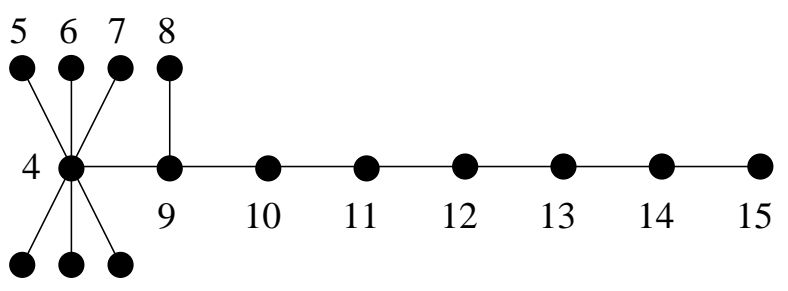

$\begin{array}{lll}1 & 2 & 3\end{array}$

Figure 1: Examples for (a) a line network, (b) non-hub target, (c) responses of a sophisticated firm $B$, and (d) not a highest central target. 
(a)

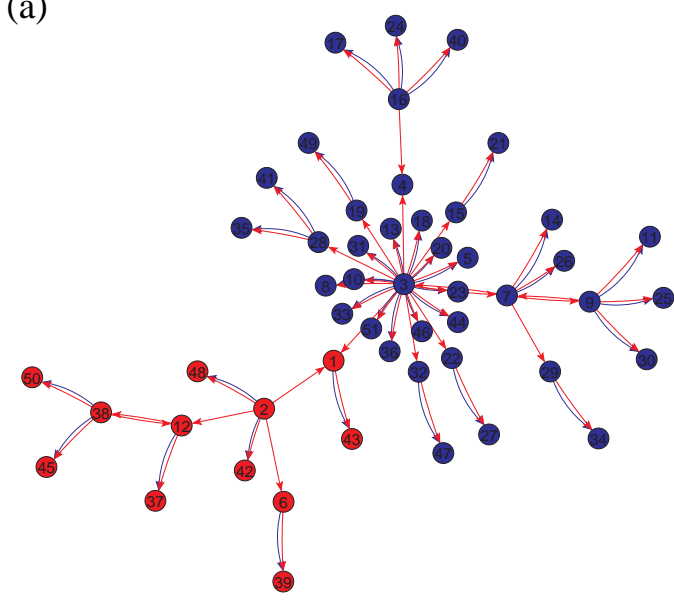

(c)

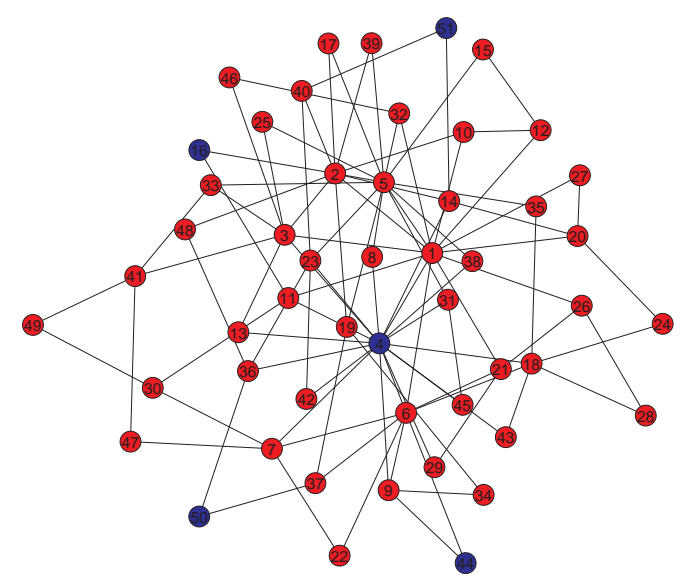

(b)

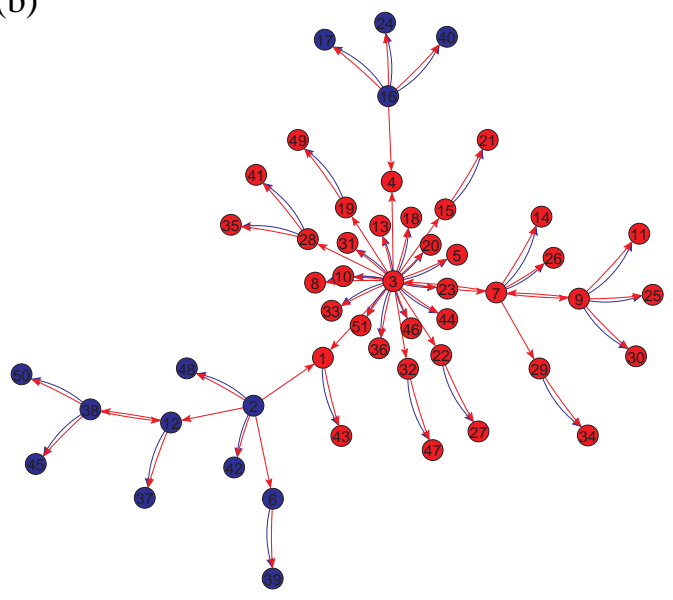

(d)

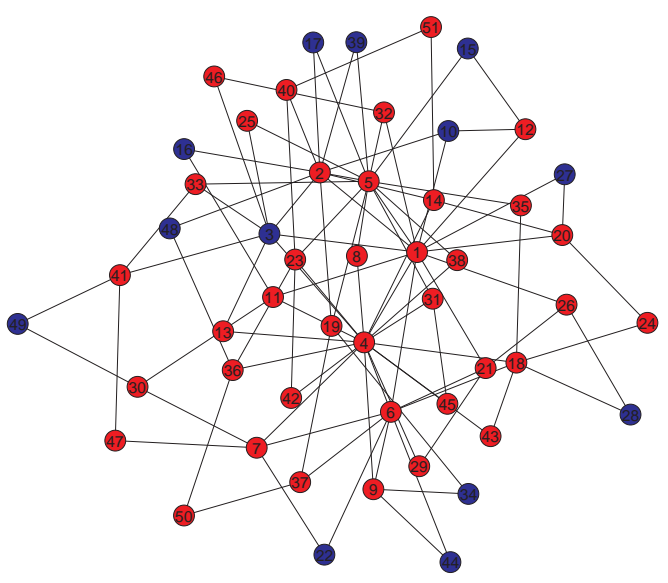

Figure 2: Simulation results on the Barabási-Albert scale-free networks with $N=51$ when $b / a=1.4$. Blue and red nodes denote $A$ and $B$ technologies, respectively. Only for the tree, blue and red arrows denote the invasion direction of $A$ and $B$, respectively. (a) For myopic firms, $\left(i_{A}, i_{B}\right)=(3,1)$ results in $\left(N^{A}, N^{B}\right)=(39,12)$. (b) For sophisticated firms, $\left(i_{A}, i_{B}\right)=(3,7)$ results in $\left(N^{A}, N^{B}\right)=(14,37)$. (c) For myopic firms, $\left(i_{A}, i_{B}\right)=(4,1)$ results in $\left(N^{A}, N^{B}\right)=(17.91,33.09)$. (d) For sophisticated firms, $\left(i_{A}, i_{B}\right)=(1,4)$ results in $\left(N^{A}, N^{B}\right)=(19.89,31.11)$. The final market shares were averaged over $10^{3}$ realizations of imitation dynamics. The networks have been produced with the PAJEK software. 

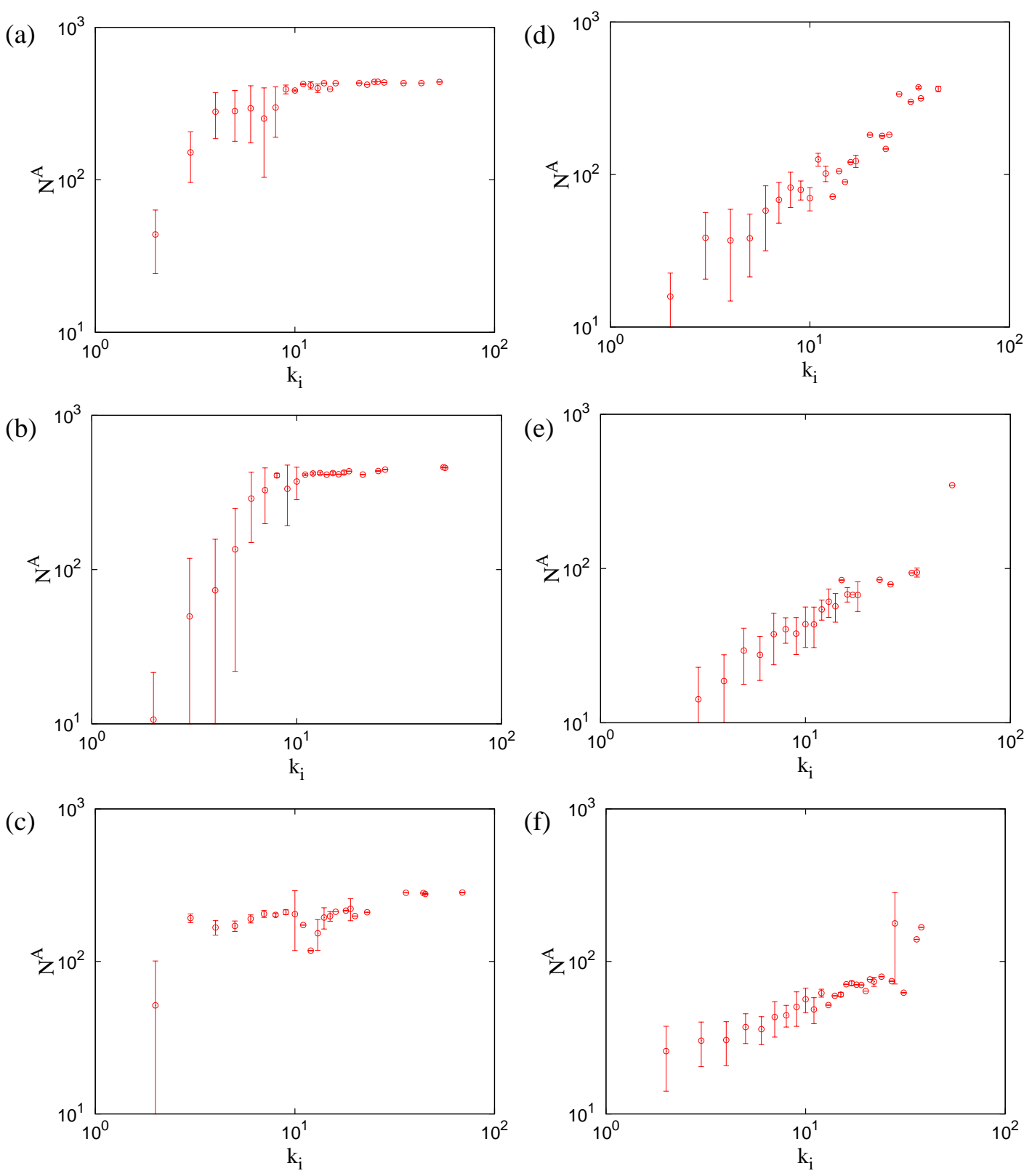

Figure 3: The correlations of the degree of $A$ 's target, $k_{i}$, and the $A$ 's resulting market share, $N^{A}$, in case of sophisticated firms. We used (a)-(c) $b / a=0.6$ and (d)-(f) $b / a=1.4$ for $r=0.3$ (top), 0 (middle), and -0.3 (bottom). We used a scale-free network with $N=500$ for each value of $r$. 


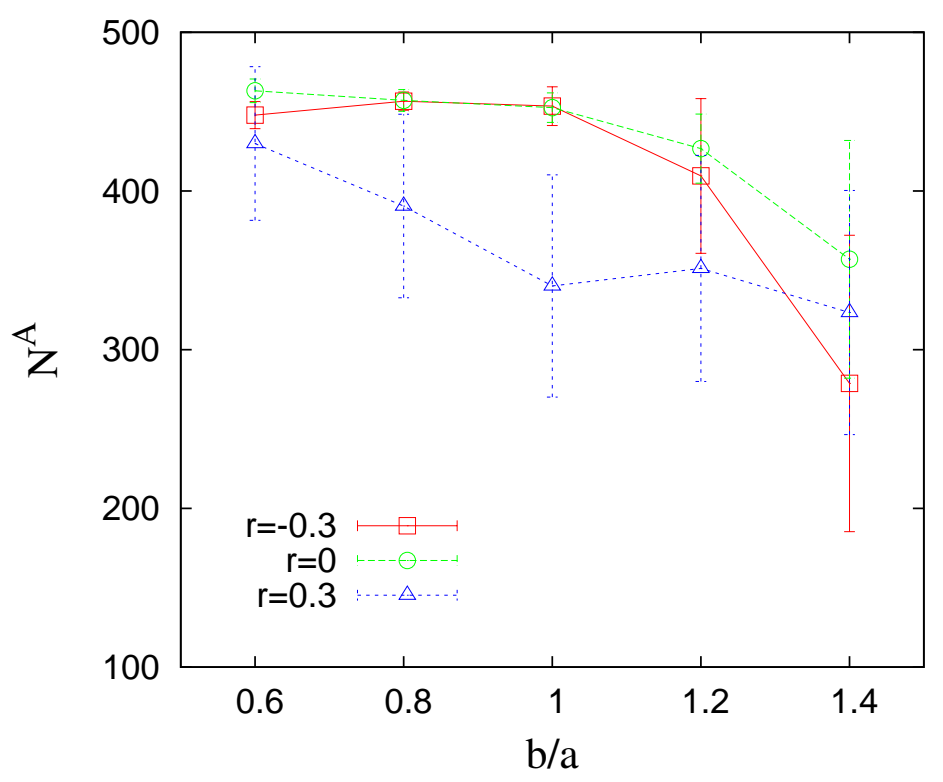

Figure 4: The dependence of $N^{A}$ on the ratio $b / a$ for the sophisticated firms on the BarabásiAlbert scale-free networks with $N=500$ over 20 different realizations of networks. 

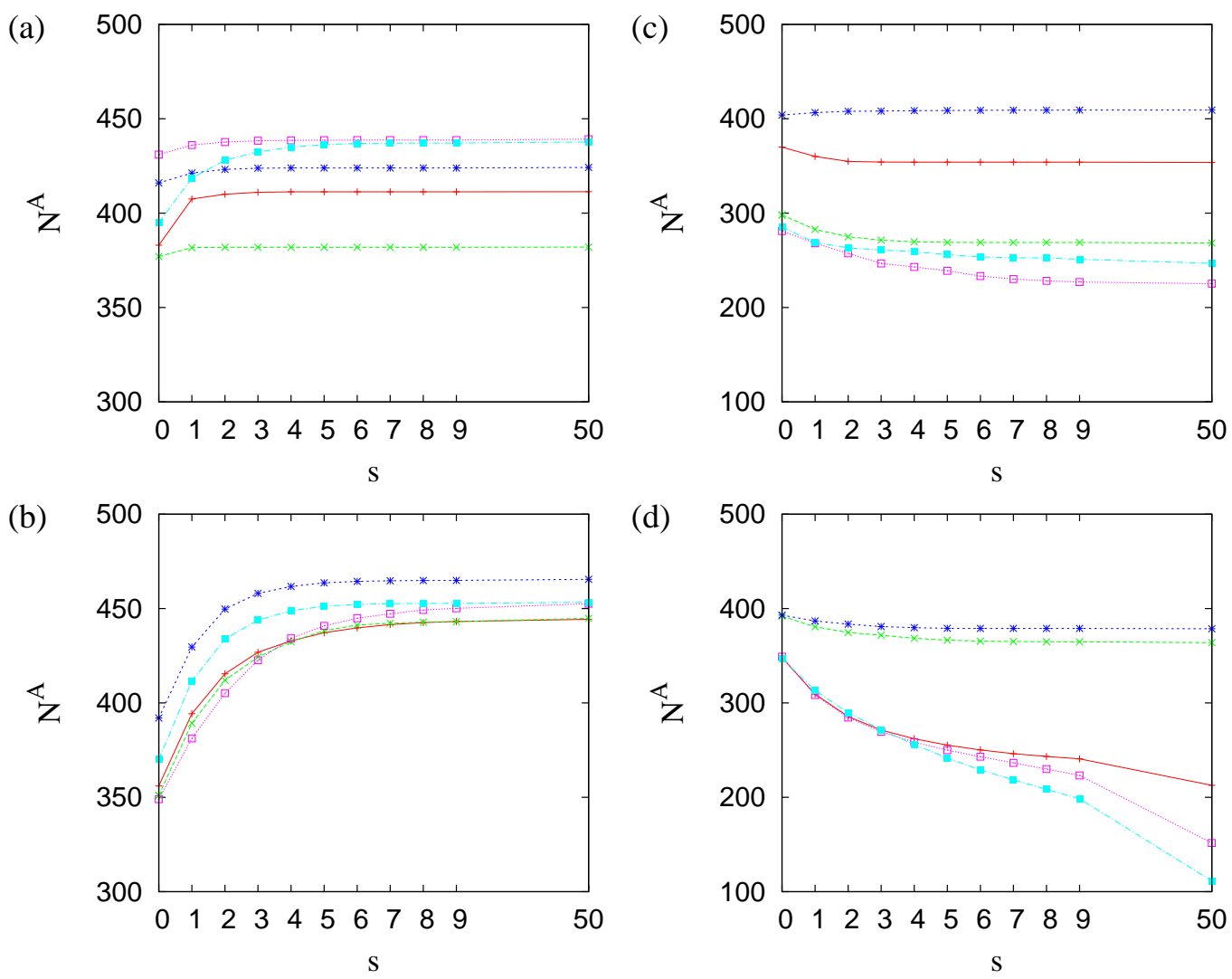

Figure 5: The dependence of $N^{A}$ on the degree of sophistication $s$ when (a)-(b) $b / a=1$ and (c)-(d) $b / a=1.4$ for $r=0.3$ (top) and -0.3 (bottom). We used the Barabási-Albert scale-free networks with $N=500$ over 5 different realizations of networks (represented by different colors). Each point was averaged over 50 realizations of imitation dynamics. $s=0$ and $s=50$ represent the myopic case and the sophisticated case, respectively. 